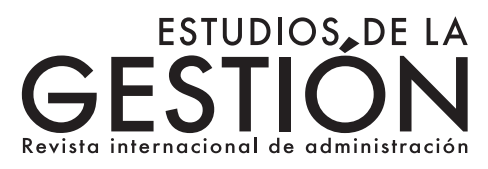

\title{
La responsabilidad social universitaria en Ecuador
}

\author{
Genoveva Espinoza Santeli \\ Universidad Andina Simón Bolívar, Sede Ecuador \\ genoveva.espinoza@uasb.edu.ec \\ Marco Guachamín Montoya \\ Universidad Andina Simón Bolivar, Sede Ecuador \\ mpato_ec@hotmail.com
}

Fecha de presentación: 5 de enero de 2015 • Fecha de aceptación: 3 de septiembre de 2015

Artículo de investigación 


\section{Resumen}

El presente trabajo analiza los supuestos teóricos y las prácticas de responsabilidad social universitaria (RSU) en las universidades ubicadas en el Distrito Metropolitano Quito (DMQ), para desarrollar un contraste entre la teoría y la práctica de la RSU al identificar los comportamientos más comunes de su gestión en los institutos de educación superior analizados.

En un primer momento se identifican las universidades, se realiza una revisión documental bibliográfica, se identifican las variables del estudio con que se hicieron encuestas, entrevistas y observación para luego proceder a la elaboración de las matrices comparativas y su correspondiente análisis.

Palabras clave: responsabilidad social universitaria, Ecuador.

JEL: M14 Cultura corporativa, responsabilidad social corporativa; I23 Centros de enseñanza superior y de investigación.

\section{Asbtract}

This paper of this paper analyzes the theoretical assumptions and practices of university social responsibility (USR) in universities located in Quito Metropolitan District (QMD), to develop a contrast between theory and practice of the RSU and identify the most common behaviors of its management.

It starts identifying the universities to be evaluated, a literature review is done. In a second moment, we identify the variables to be used during the survey, interviews and observations. Finally, a comparison between the universities followed.

Keywords: university social responsibility, Ecuador.

JEL: M14 Corporate culture, corporate social responsibility; I23 Higher education and research.

\section{Resumo}

Este artigo analisa os pressupostos e práticas de responsabilidade social universitária (USR) em universidades do Distrito Metropolitano de Quito (DMQ) teóricas, para desenvolver um contraste entre a teoria ea prática do RSU para identificar os comportamentos mais comum de sua gestão nos colégios analisados.

$\mathrm{O}$ autor começa a identificar as universidades, numa revisão de documentos é feito, as variáveis de estudo em que pesquisas, entrevistas e observações foram feitas e, em seguida, avance para o desenvolvimento de matrizes comparativas foram identificados.

Palavras-chave: responsabilidade social da universidade, Equador.

JEL: M14 Cultura corporativa, responsabilidade social corporativa; I23 Educação superior e da investigação. 


\section{Introducción}

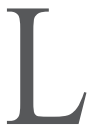

a universidad es un actor transcendental en la sociedad, tiene como principal función educar, formar y convertir a los ciudadanos en profesionales a través de la generación y transmisión de conocimientos; al mismo tiempo, la universidad ejerce una gran influencia en el desarrollo y crecimiento de un país; esta formación no solo debe proveer de conocimientos, doctrinas y teorías; debe ser impartida con una visión ética humanista y comunitaria, que genere un impacto positivo a nivel individual y colectivo.

La responsabilidad social, en una perspectiva ética aplicada a la empresa, defiende que la cultura afecta la toma de decisiones a través de las evaluaciones deontológicas y teológicas del individuo (Vitell y Paolillo 2004) o desde la teoría del bien común, que es entendido como un valor universal que compete a la comunidad en general y no solo a un individuo (Smith 2001). Esa responsabilidad ha sido acogida principalmente por las empresas que han tomado conciencia y se han hecho responsables de los impactos negativos que sus actividades ocasionan en sus grupos de interés. Esta idea de hacerse responsable por su comportamiento y considerar la esfera de influencia, en el momento de tomar decisiones o ejecutar nuevos proyectos, ha evolucionado hacia otras esferas y la sociedad actual tiene la expectativa de que no solo la empresa sea socialmente responsable, sino también organizaciones públicas y privadas, sindicatos, fundaciones, empresas mixtas e instituciones educativas primarias, secundarias y superiores, por nombrar algunas. Las universidades están adoptando e incorporando en sus actividades el concepto de responsabilidad social universitaria.

La responsabilidad social universitaria (RSU) es una política de calidad ética del desempeño de la comunidad universitaria a través de la gestión responsable de los impactos educativos, cognitivos, laborales, sociales y ambientales que la universidad genera, en un diálogo participativo con la sociedad para promover el desarrollo humano sustentable. 
El estudio de la RSU enfrenta varias limitaciones: no existen estadísticas o información relevante sobre el quehacer universitario con respecto a su responsabilidad social; las universidades ecuatorianas todavía no cuentan con un departamento o instancia que gestione directamente la RSU, lo que genera un desconocimiento generalizado sobre los conceptos y las prácticas de responsabilidad social universitaria.

Considerando la limitada información específica que se cuenta sobre la RSU en el Ecuador, la presente investigación pretende realizar una caracterización de las prácticas de RSU en las universidades del DMQ.

\section{La responsabilidad social universitaria}

En términos generales, se puede conceptualizar la responsabilidad social empresarial (RSE) como un compromiso voluntario adquirido por las organizaciones con el fin de mejorar la calidad de vida de sus stakeholders, o grupos de interés, a partir de la implementación de estrategias, iniciativas y políticas que busquen mejorar los rendimientos económicos al mismo tiempo que buscan una mejor inclusión y justicia social a través de la creación de empleo, respeto a los derechos humanos y a las prácticas laborales, considerando los límites de los ecosistemas naturales.

La responsabilidad social universitaria es un concepto en construcción en el cual la universidad y las instituciones de educación superior son el principal actor que, en el momento se encuentran desarrollando un proyecto de promoción social, con principios éticos alineados al desarrollo sustentable y a la responsabilidad social, en la cual buscan que el conocimiento que imparten esté enmarcado en un ambiente de respeto, responsabilidad, igualdad e inclusión. Sin embargo, existen sectores que entienden la RSU únicamente como la ejecución de proyectos enfocados a extensión universitaria, proyección social, voluntariado estudiantil, servicio estudiantil universitario, entre otros; en realidad, la RSU engloba más aspectos del compromiso que tienen las universidades con la sociedad.

La Organización de las Naciones Unidas para la Educación, la Ciencia y la Cultura (UNESCO por sus siglas en inglés), a partir de sus conferencias mundiales de educación superior y su principio de pertinencia de la educa- 
ción superior, visibilizó la necesidad de incorporar en la agenda la responsabilidad social universitaria en la producción y difusión de conocimientos. (UNESCO 1998). De acuerdo con Gaete $(2014,105)$ los orígenes de la RSE "parecen estar relacionados con los efectos de los cambios sociales en la educación superior acaecidos durante la década de los setenta, entre otros el acceso menos elitista a las universidades y la revalorización del conocimiento para la sociedad y la economía".

Una de las iniciativas globales e importantes para la ciudadanía corporativa y la RSE es el Pacto Global de las Naciones Unidas (1999) que cuenta con un decálogo de principios divididos en cuatro áreas: derechos humanos, prácticas laborales, anticorrupción y medioambiente. Las Naciones Unidas, a través de la oficina del Pacto Global, considerando la importancia que tiene la educación superior y las recomendaciones de la UNESCO, en 2007 desarrollaron una herramienta específica para RSU, los Principios para la Educación Responsable en Gestión (PRME por sus siglas en inglés), una plataforma de participación voluntaria principalmente desarrollada para las escuelas de negocios y los programas de gestión. Los institutos de educación superior, al adherirse a los PRME, expresan su compromiso de integrar los valores universales en su malla curricular y en sus líneas de investigación, "con el objetivo de contribuir al mercado global y a la construcción de sociedades más prosperas" (Pacto Global 2007a, 5). Los seis principios que recoge la plataforma son: propósito, valores, método, investigación, paternariado y diálogo, a través de los cuales se proporciona un instrumento para que las universidades integren la sostenibilidad corporativa y la investigación en las prácticas de la educación (Pacto Global 2007b).

La RSU no puede quedarse en la dimensión tradicionalmente conocida como extensión universitaria; tampoco debe ser entendida dentro de los parámetros de la RSE; debe buscar garantizar la calidad de la educación superior a través del fortalecimiento y adaptación al entorno académico y la realidad actual. Por ello no puede pasar por alto: formar profesionales con conocimientos pertinentes a los requerimientos del entorno, sensibles y motivados por valores; orientar la investigación científica hacia la solución de problemas sociales; desarrollar proyectos con impacto social real; propiciar la transferencia de conocimiento y tecnología a la sociedad; interactuar y dialogar con la sociedad; capacitar al más alto nivel a sus profesores y empleados; apoyar el voluntariado estudiantil; y concienciar a los alumnos 
sobre la corresponsabilidad de todos en la solución de los problemas del mundo (González y Túñez 2014).

De acuerdo con el Reglamento de Carrera y Escalafón del Profesor Investigador del Sistema de Educación Superior (2012) y la Ley Orgánica de Educación Superior (2010), las actividades principales de las universidades y sus docentes son cuatro: la docencia, investigación, vinculación con la sociedad y gestión académica, y es en todas estas áreas que se debe incluir la perspectiva de la RSU. Para Remolina (2003) las universidades deben prestar servicios profesionales bajo criterios éticos y humanísticos, de solidaridad y excelencia en el servicio, por lo que las universidades cada día están obligadas por la sociedad para que apliquen principios de responsabilidad social en los diferentes procesos clave: formación, investigación y extensión. Según Aguirre, Pelekais y Paz $(2012,15)$, las universidades "deben generar la responsabilidad social como compromiso institucional, no como un indicador enmarcado en los principios políticos gubernamentales, que se deriva de la acción y actitud estratégica voluntaria con su gente, para asegurar la competitividad de hechos valorativos significativos, permitiendo intercambiar ideas, comportamientos, la integración de valores e internalización de las necesidades sociales".

Desde una visión integral, la RSU se refiere al área de extensión y proyección social, es decir, a toda la institución en su conjunto, siendo uno de los valores más importantes la coherencia institucional, la coincidencia entre la acción y el discurso institucional (Vallaeys y Carrizo 2006). El mismo autor señala que la RSU reintegra la función social universitaria en cuatro procesos sustantivos: gestión, formación, producción de conocimientos y participación social (Vallaeys 2009), procesos que están plenamente alineados con la legislación ecuatoriana en cuanto al quehacer universitario se refiere.

El Banco Internacional de Desarrollo ha promovido el uso del Manual de Vallaeys, De la Cruz y Sasia (2009) para que las universidades latinoamericanas den sus primeros pasos en la implementación de políticas de RSU, motivo por el cual se ha decidido usar dicho Manual en la metodología de investigación del presente trabajo.

Vallaeys (2010) define a la RSU como:

Una política de calidad ética del desempeño de la comunidad universitaria (estudiantes, docentes y personal administrativo) a través de la gestión responsables de los impactos 
educativos, cognitivos, laborales, sociales y ambientales que la universidad genera, en un diálogo participativo con la sociedad para promover el Desarrollo Humano Sostenible.

El concepto es amplio y abarca todo el ámbito universitario: la investigación, la docencia, el personal administrativo y todos los servicios universitarios y, además, engloba la gestión de impactos que genera la universidad a sus stakeholders: impactos organizacionales, educativos, cognitivos y epistemológicos y sociales.

Los impactos generados por la universidad influyen directamente en los ámbitos de acción y al incorporar variables de medición se podrán determinar las prácticas de RSU realizadas en las universidades seleccionadas y comparar con el modelo propuesto por Vallaeys en su manual. Los ámbitos que se analizaron, considerando que todos implican la gestión socialmente responsable de la universidad, son:

1. Campus responsable: procedimientos, clima laboral, recursos humanos, gestión interna y el cuidado y beneficio del medioambiente, considerando a todos los stakeholders de la universidad.

2. Gestión social del conocimiento: difusión del saber, investigación y formación de modelos de producción de conocimiento que orienten a la actividad de investigación científica y que generen desarrollo local y nacional en el cual se incluya el principio de materialidad de todos los grupos de interés.

3. Participación social: gestión de la universidad en la comunidad, mediante la realización de proyectos sociales consensuados con los actores de la sociedad para el aprendizaje en conjunto y el desarrollo social de soluciones integrales.

4. Formación profesional y ciudadana: formación académica en lo relativo a la temática, organización curricular metodología y propuesta didáctica.

En concordancia con lo anteriormente descrito, la responsabilidad social universitaria debe formar parte de las universidades con el entorno y la sociedad, siendo fundamental el compromiso con las políticas y los propósitos que hemos analizado. De esta forma será posible mejorar el ambiente universitario, fomentar el desarrollo sustentable del entorno y aumentar la calidad en el sistema universitario actual. El compromiso de las universidades con la responsabilidad social universitaria les permite compensar a la sociedad lo 
que reciben de ella, contribuyendo con profesionales éticamente responsables que fomenten un mayor equilibrio social y humano.

\section{Metodología}

Para el presente trabajo se utilizó una investigación no experimental transversal descriptiva; como herramienta se aplicó el Manual de la Responsabilidad Social Universitaria desarrollado por Vallaeys (2009) y recomendado por el BID sobre el diagnóstico de efectividad de la relación de la universidad con la sociedad, como proceso de mejora continua de la universidad a partir de los impactos identificados. Los ámbitos analizados fueron:

Tabla 1

Dimensiones de evaluación universitaria

\begin{tabular}{|l|l|}
\hline \multicolumn{1}{|c|}{ Ámbito } & \multicolumn{1}{c|}{ Dimensiones } \\
\hline Organizacional & $\begin{array}{l}\text { En tanto institución que opera en torno a un proyecto universitario, con una } \\
\text { estructura que lo desarrolla y unas políticas concretas que lo promueven. }\end{array}$ \\
\hline Educativo & $\begin{array}{l}\text { En tanto institución que se encarga de la formación de sus estudiantes, con } \\
\text { una vocación profesional y cívica. }\end{array}$ \\
\hline Del conocimiento & En tanto institución que investiga, que produce saber y lo transmite. \\
\hline Ámbito social & $\begin{array}{l}\text { En tanto institución que forma parte de la sociedad e interactúa con otros } \\
\text { agentes, colectivos y comunidades, tanto a nivel local como global. }\end{array}$ \\
\hline
\end{tabular}

Fuente: Vallaeys 2009.

Elaboración propia.

Las etapas fueron: revisión bibliográfica, revisión documental, investigación de campo a través de entrevistas en profundidad cualitativas, revisión de las páginas web de las instituciones para determinar la información que se está reportando a sus grupos de interés con relación a la RSU y análisis de los resultados obtenidos.

La revisión bibliográfica consistió en un levantamiento de los principales conceptos sobre el tema central, es decir, la responsabilidad social universitaria. Se realizó una búsqueda exhaustiva del tema en las siguientes bases 
de datos: Jstor, Ebsco, Proquest, Hapi, y Springer; se usó la combinación de las palabras "responsabilidad social universitaria" y "responsabilidad social universitaria" y "Ecuador", y se obtuvieron los siguientes resultados:

Tabla 2

Resultado de búsquedas en bases de datos

\begin{tabular}{|l|c|c|c|}
\hline $\begin{array}{c}\text { Base de } \\
\text { datos }\end{array}$ & $\begin{array}{c}\text { Número de artícu- } \\
\text { los con "respon- } \\
\text { sabilidad social } \\
\text { universitaria" }\end{array}$ & $\begin{array}{c}\text { Número de artículos } \\
\text { con "responsabilidad } \\
\text { social universitaria" + } \\
\text { Ecuador }\end{array}$ & $\begin{array}{c}\text { Número de artículos con } \\
\text { "responsabilidad social } \\
\text { universitaria" + materia } \\
\text { "social responsibility" }\end{array}$ \\
\hline Jstor & 0 & 0 & 0 \\
\hline Ebsco & 53 & 1 & 11 \\
\hline Proquest & 20 & 9 & 19 \\
\hline Hapi & 1 & 0 & 0 \\
\hline Springer & 1 & 0 & 0 \\
\hline
\end{tabular}

Fuente: Bases de datos Universidad Andina Simón Bolívar.

Elaboración propia.

De estos resultados se analizaron todos los artículos que cumplían con los requisitos de "responsabilidad social universitaria" dentro de la materia de "social responsibility", con los cuales se construyó el marco teórico y se establecieron las categorías para el análisis en las universidades seleccionadas.

Para identificar las universidades evaluadas se usó el listado de categorización de las universidades acreditadas por el Consejo de Evaluación, Acreditación y Aseguramiento de la Calidad de la Educación Superior (CEAACES), ${ }^{1}$ año 2013. El universo de universidades fue categorizado considerando si estas ofertan pregrado y posgrado, solo pregrado o solo o solo posgrado; el CEAACES categorizó a las universidades en 4 categorías: A, B, $\mathrm{C}$ y $\mathrm{D}$, tomando en cuenta una serie de indicadores que consideran la produc-

1. Es un organismo técnico, público y autónomo encargado de ejercer la rectoría política para la evaluación, acreditación y el aseguramiento de la calidad de las Instituciones de Educación Superior, sus programas y carreras. Realizan procesos continuos de evaluación y acreditación que evidencien el cumplimiento de las misiones, fines y objetivos de las universidades. 
ción científica, el número de docentes contratados, el número de alumnos, infraestructura, condiciones laborales de los docentes, entre otros.

Tabla 3

Número de universidades por categoría

\begin{tabular}{|l|c|c|c|c|}
\hline & $\begin{array}{c}\text { Categoría } \\
\text { A }\end{array}$ & $\begin{array}{c}\text { Categoría } \\
\text { B }\end{array}$ & $\begin{array}{c}\text { Categoría } \\
\text { C }\end{array}$ & $\begin{array}{c}\text { Categoría } \\
\text { D }\end{array}$ \\
\hline Oferta académica de pregrado y posgrado & 3 & 18 & 14 & 6 \\
\hline Oferta académica de pregrado & 0 & 4 & 4 & 2 \\
\hline Oferta académica de posgrado & 2 & 1 & 0 & 0 \\
\hline
\end{tabular}

Fuente: CEAACES 2013.

Elaboración propia.

Para la presente investigación se consideraron las instituciones educativas con oferta académica de pregrado y posgrado catalogados en la Categoría A que tienen su domicilio principal en el DMQ, por lo que la investigación fue realizada en dos universidades que cumplen con los requisitos antes mencionados: Escuela Politécnica Nacional (EPN) y Universidad San Francisco de Quito (USFQ). La tercera universidad que está en esta categoría es la Escuela Superior Politécnica del Litoral con domicilio en la ciudad de Guayaquil, motivo por el cual no fue considerada en el presente estudio.

La revisión documental complementó la primera etapa aportando la construcción de una matriz de variables, con un sistema de medición del suceso, para las universidades seleccionadas. La investigación de campo a través de las entrevistas cualitativas confirmó los hallazgos obtenidos en las dos etapas anteriores. Finalmente, con los resultados obtenidos, se realizó un análisis de resultado con la información levanta de las universidades analizadas.

\section{Resultados}

La revisión de la literatura dio como resultado que el tema de la responsabilidad social universitaria es relativamente nuevo para las universidades latinoamericanas: casi no existen artículos académicos sobre la materia. Se 
analizaron todos los artículos que están en las bases de datos mencionadas en la metodología y la mayoría de los artículos usan la metodología propuesta por Vallaeys o crean sus propios sistemas de gestión para la RSU. Con el análisis de la información bibliográfica se tomó en consideración la declaración o verificación de temas elementales para una adecuada gestión de responsabilidad social universitaria que son presentadas en el siguiente cuadro comparativo:

Tabla 4

Comparativo de la revisión documental de las universidades evaluadas

\begin{tabular}{|l|l|c|c|}
\hline \multicolumn{1}{|c|}{ Tema } & \multicolumn{1}{|c|}{ Categoría } & EPN & USFQ \\
\hline Identidad & Declaran: principios, misión, visón, valores. & Todos & Todos \\
\hline $\begin{array}{l}\text { Vinculación con la } \\
\text { comunidad }\end{array}$ & $\begin{array}{l}\text { Declaran: extensión universitaria, trabajo } \\
\text { social, programas o proyectos. }\end{array}$ & Todos & Todos \\
\hline $\begin{array}{l}\text { Transparencia } \\
\text { de la información }\end{array}$ & $\begin{array}{l}\text { Declaran: informes financieros, balance } \\
\text { económico. }\end{array}$ & Ninguno & Todos \\
\hline $\begin{array}{l}\text { Medios } \\
\text { de difusión }\end{array}$ & $\begin{array}{l}\text { Declaran: herramientas de comunicación, } \\
\text { revistas científicas, anuarios, boletines, página } \\
\text { web, facebook, twitter, etcétera. }\end{array}$ & Todos & Todos \\
\hline
\end{tabular}

Fuente: los autores.

Elaboración propia.

Las dos universidades presentan una gran similitud en los temas de identidad, vinculación con la comunidad, programas de beneficio social y medios de difusión de información básica al público en general, pese a que las universidades seleccionadas, EPN y USFQ, son de financiamiento público y privado, respectivamente.

El tema en que difieren, y que requiere ser destacado, es la transparencia de la información; uno de los principios básicos de la RSE, y por tanto de la RSU, es la apertura que tienen las organizaciones para informar y transparentar fidedignamente; es el acceso a los datos, documentos e informes. De acuerdo con la Asociación Española de Contabilidad y Administración (2004), la transparencia es uno de los principales atributos y un principio básico de la responsabilidad social corporativa; en palabras de Lizcano (2004), 
si las organizaciones son poco transparentes no están asumiendo el concepto ni los objetivos de la RSE, por lo que es indispensable que las universidades informen a sus grupos de interés todas sus actividades. La USFQ mantiene toda su información financiera y económica a disposición del público en general; en el caso de la EPN, para poder acceder a su información económica, es necesario realizar un pedido formal a las instancias administrativas para analizar la posibilidad de obtener una copia de la información, es decir, no cumplen con el principio de transparencia requerido por la RSU.

El tema fundamental analizado en la revisión documental tiene relación con las variables de estudio debido a que, a partir de las mismas, se desarrolló el sistema de matrices de cada universidad analizada. Las variables de estudio tienen como finalidad la descripción de las principales prácticas de RSU que se desarrollan internamente en las universidades analizadas. La construcción de la matriz está conformada por las siguientes variables: campus responsable, gestión social del conocimiento, participación social y formación profesional y ciudadana, coherente con un sistema de medición para las dos universidades seleccionadas.

Sobre la base de cada impacto de la RSU se desarrollaron temas generales que sirvieron de herramienta para la explicación y comprobación de la existencia de los impactos analizados. Las variables se detallan en la tabla 5:

Tabla 5

Variables de estudio

\begin{tabular}{|c|l|}
\hline Variable & \multicolumn{1}{c|}{ Tema } \\
\hline \multirow{4}{*}{ Campus responsable } & Derechos humanos, equidad de género y no discriminación. \\
\cline { 2 - 2 } & $\begin{array}{l}\text { Desarrollo personal y profesional, buen clima de trabajo y aplicación de } \\
\text { los derechos laborales. }\end{array}$ \\
\cline { 2 - 2 } & Medioambiente (campus sostenible). \\
\cline { 2 - 2 } & Transparencia y democracia (buen gobierno). \\
\cline { 2 - 2 } & Comunicación y marketing responsable. \\
\hline
\end{tabular}




\begin{tabular}{|l|l|}
\hline \multirow{5}{*}{$\begin{array}{l}\text { Gestión social } \\
\text { del conocimiento }\end{array}$} & $\begin{array}{l}\text { Promoción de equipos interdisciplinarios (cooperación varias } \\
\text { disciplinas) y transdisciplinarios (conocimientos que trascienden } \\
\text { las disciplinas). }\end{array}$ \\
\cline { 2 - 2 } & $\begin{array}{l}\text { Integración de actores sociales externos en las investigaciones y } \\
\text { el diseño de líneas de investigación. }\end{array}$ \\
\cline { 2 - 3 } Participación social & $\begin{array}{l}\text { Difusión y transferencia de conocimientos socialmente útiles hacia } \\
\text { públicos desfavorecidos. }\end{array}$ \\
\cline { 2 - 2 } & Promoción de investigaciones aplicadas a temas de desarrollo. \\
\hline \multirow{5}{*}{$\begin{array}{l}\text { Formación } \\
\text { profesional }\end{array}$} & Integración de la formación académica con la proyección social. \\
\cline { 2 - 3 } y ciudadana & Evaluación del servicio universitario a la comunidad. \\
\cline { 2 - 3 } & Promoción de redes sociales para el desarrollo. \\
\hline Participación activa en la agenda local y nacional de desarrollo. \\
\hline
\end{tabular}

Fuente: adaptación de Vallaeys, De la Cruz y Sasia 2009.

Elaboración propia.

Sobre las variables descritas se generó una matriz de presencia de los fenómenos en la universidad, con preguntas asociadas a cada tema, otorgándoles un peso relativo u ocurrencia del hecho en una escala tipo Likert ${ }^{2}$ que califica la observancia del fenómeno en escalas ordinarias que van de 10 a 0 y que representan el grado de existencia del fenómeno en alta, media, baja o inexistente (Nogales 2004).

2. La escala de Likert es un tipo de instrumento de medición o de recolección de datos que se dispone en la investigación social para medir actitudes. Permite medir la actitud hacia un objeto a base del grado de acuerdo o desacuerdo, alto o bajo, de los entrevistados con una serie de enunciados positivos o negativos que representan atributos del objeto estudiado. 
Tabla 6

Escala de ocurrencia

\begin{tabular}{|l|l|}
\hline Ocurrencia & Resultado \\
\hline De 1 a 2 & Inexistente \\
\hline De 3 a 4 & Baja \\
\hline De 5 a 6 & Media \\
\hline De 7 a 8 & Media alta \\
\hline De 9 a 10 & Alta \\
\hline
\end{tabular}

Fuente: los autores.

Elaboración propia.

La calificación de la ocurrencia del fenómeno está en función de la existencia del suceso a través de indicadores construidos a base de los temas globales de cada impacto de la RSU y su observancia en las universidades seleccionadas cuyos resultados finales son:

Tabla 7

\section{Comparativo de revisión documental}

\begin{tabular}{|l|l|l|}
\hline \multicolumn{1}{|c|}{ Variable } & \multicolumn{1}{|c|}{ EPN } & \multicolumn{1}{c|}{ USFQ } \\
\hline Campus responsable & Media & Media alta \\
\hline Formación profesional y ciudadana & Media alta & Media \\
\hline Gestión social del conocimiento & Media & Media alta \\
\hline Participación social & Media & Media alta \\
\hline
\end{tabular}

Fuente: los autores.

Elaboración propia.

Para los autores, la variable de mayor importancia es la formación profesional y ciudadana, por su impacto en la sociedad. De las universidades analizadas se puede destacar que, por su modelo de enseñanza de artes liberales, la USFQ tiene materias en programas regulares que promulgan el desarrollo 
sustentable, responsabilidad social universitaria en concordancia con el desarrollo profesional con metodologías de fácil aprendizaje con colaboración de sus clientes internos y externos. En el caso de la EPN en la que su modelo de enseñanza es tradicional, fundamentado principalmente en el desarrollo profesional de sus estudiantes, el resultado son profesionales muy capacitados en áreas científicas y teóricas, pero con un limitado conocimiento de la responsabilidad social de sus acciones en el medio.

Otro tema de importancia es la gestión social del conocimiento, ya que tiene un impacto directo sobre la producción y difusión de los conocimientos adquiridos a la sociedad; las dos universidades gestionan y generan profesionales de alto nivel, pero la difusión de sus conocimientos expresados a través de revistas de actualidad, ciencia, simposios, anuarios o foros de discusión, son difundidos únicamente a instituciones como el gobierno, los municipios y las universidades, pero con poca interacción con la sociedad.

La participación social entendida como una variable de gestión de responsabilidad social es un nexo que mantienen las dos universidades aunque en menor grado, básicamente porque la estructura de enseñanza se basa en la formación de nuevos profesionales y su vinculación se genera con el aporte de nuevos profesionales que van a mejorar el estándar de vida de sus familias y en un futuro el desarrollo de la sociedad.

La investigación de campo, que se llevó a cabo a través de entrevistas cualitativas a personas encargadas de gestionar directamente las variables de estudio, confirmó los resultados obtenidos en las dos etapas anteriores. Las personas entrevistadas en las dos universidades concuerdan en que la formación de los estudiantes en su mayoría está encaminada al conocimiento científico teórico, con materias regulares que no contienen aspectos fundamentales de la responsabilidad social, lo que conlleva a un desconocimiento de la gestión socialmente responsable de los estudiantes y profesores con la sociedad en general.

No se evidenció, en las entrevistas realizadas, el interés por las universidades para la creación de un departamento dedicado a la gestión de la RSU, debido principalmente al desconocimiento de la temática de la responsabilidad social universitaria en los dos establecimientos de educación superior. 


\section{Conclusiones}

- La responsabilidad social universitaria es la gestión socialmente responsable de los impactos que la universidad genera en la sociedad y en todos sus stakeholders.

- La RSU debe ser investigada, implementada y evaluada por todos los docentes, investigadores, estudiantes y administrativos de una universidad para incorporar en sus actividades diarias un nuevo paradigma de concepción social, optimizando todos sus esfuerzos y estudios en beneficio de la sociedad.

- La educación superior es un derecho de toda la sociedad que busca formar profesionales alineados en la justicia, la equidad y el desarrollo sostenible; en este sentido la RSU debe ser intrínseca al quehacer universitario.

- La RSU no debe ser entendida únicamente como una extensión universitaria o voluntariado estudiantil, su propósito fundamental es la formación de nuevos profesionales éticos y socialmente responsables con todos sus grupos de interés.

- El principal aporte de la RSU es la gestión de los impactos que genera en la sociedad, en los temas: formación ética y socialmente responsable de nuevos profesionales; producción y difusión de conocimientos y en el desarrollo de proyectos sociales que vinculen a los estudiantes a la realidad del país; consecuentemente, todo el sector productivo se desarrolla en función del ámbito social.

- Las universidades no mantienen información actualizada y dedicada para temas de RSU, lo que ocasiona desconocimiento a sus clientes internos y externos de los beneficios de la gestión socialmente responsable.

- La gestión de docencia fundamentada en el modelo de artes liberales practicado por la USFQ marca la diferencia en el aprendizaje de los saberes, ya que amplía la concepción de la realidad del entorno al encasillar a los estudiantes a una malla educativa preestablecida, con materias técnicas y teóricas, dejando de lado el estudio de temas sociales.

- El estudio comparativo de las variables que fundamentan la RSU en las universidades analizadas evidenció la mayor ocurrencia de las siguientes prácticas: 
- La responsabilidad de las universidades radica principalmente en la formación teórico-científica de nuevos profesionales, pero carentes de conocimientos de responsabilidad social universitaria o de proyectos sociales integrales.

- Las políticas, las normas y los reglamentos se gestionan eficientemente al interior de las universidades; sin embargo, al no poseer conocimientos explícitos de la RSU, no constan en sus escritos informativos, ocasionando una brecha entre los lineamientos generales con respecto a nuevos procedimientos en materia social y en responsabilidad.

- A pesar de que el conocimiento de la RSU no es nuevo, las universidades estudiadas no mantienen departamentos que gestionen directamente la RSU; solo mantienen departamentos o instancias que gestionan una parte de la responsabilidad social universitaria, debido a su estructura organizativa o percepción del entorno.

- Las universidades efectúan varios proyectos de beneficio social de diversa índole (alimenticios, geológicos, médicos, técnicos, entre otros); sin embargo, las iniciativas son poco difundidas y promocionadas en sus medios, limitándose a revistas, memorias e informes de uso interno, creando una dependencia de portales web que no generan incidencia en la sociedad.

- Las universidades seleccionadas mantienen un compromiso social de colaborar en proyectos de beneficio comunitario, para lo cual generan nuevos proyectos de incidencia social, como son los centros de transferencia de conocimientos y mesas participativas de dialogo, en busca de soluciones integrales para problemas sociales.

- En conjunto, las variables analizadas en las universidades seleccionadas (campus responsable, formación profesional, gestión social del conocimiento y participación social) son una herramienta efectiva para comparar e inferir la gestión de la RSU de las universidades sin que influya el tipo de financiamiento, categoría y locación geográfica. 


\section{Genoveva Espinoza Santeli y Marco Guachamín Montoya}

\section{Referencias}

Aguirre, René, Cira Pelekais y Annherys Paz. 2012. "Responsabilidad social: compromiso u obligación universitaria”. Telos: revista de estudios interdisciplinarios en ciencias sociales 14, No. 1: 11-20.

Alvarado, Alejandro, Enrique Bigné y Rafael Currás. 2011. "Perspectivas teóricas usadas para el estudio de la responsabilidad social empresarial: una clasificación con base en su racionalidad”. Estudios Gerenciales 277, No. 118 (enero-marzo): 115-137.

Asamblea Nacional Constituyente. 2010. Ley Orgánica de Educación Superior. Quito: Registro Oficial, Suplemento, No. 298.

Asociación Española de Contabilidad y Administración de Empresas (AECA). 2004. Marco conceptual de la responsabilidad social corporativa. Madrid: AECA.

Barroso, Francisco. 2008. "La responsabilidad social empresarial: un estudio en cuarenta empresas de la ciudad de Mérida, Yucatán”. Contaduría y Administración, No. 226: 73-91.

Consejo de Educación Superior (CES). 2012. "Reglamento de carrera y escalafón del profesor e investigador del sistema de educación superior”. Quito: CES. 〈http://www.ces.gob.ec/ index.php?option=com phocadownload\&view=category\&id=12\&Itemid=496 .

Consejo de Evaluación, Acreditación y Aseguramiento de la Calidad de la Educación Superior Evaluación de universidades (CEAACES). 2013. "Categorización de universidades 2013”. Consulta: febrero de 2015. 〈http://www.ceaaces.gob.ec/sitio/evaluacion-univer sidades-2013/>.

Gaete Quezada, Ricardo. 2014. "La responsabilidad social universitaria como política pública: un estudio de caso". Documentos y Aportes en Administración Pública y Gestión Estatal. Santa Fe: Universidad Nacional del Litoral, No. 22: 103-127.

González, Karina, y José Miguel Túñez. 2014. "Responsabilidad social universitaria: apuntes para un modelo de RSU”. Revista de Comunicación, No. 13 (enero-diciembre): 84-117.

Lizcano, José Luis. 2004. "Transparencia informativa y responsabilidad social corporativa". Boletín de Estudios Económicos 59, No. 182: 279-303.

Morales, Jesús, Yanet Villanueva, María del Carmen Armenteros, Gabriela Reyna y José Duque. 2015. "La responsabilidad social universitaria en Coahuila, México: estudio exploratorio". Revista Global de Negocios 4, No. 1: 1-19.

Naval, Concepción, y Marta Ruiz-Corbella. 2012. “Aproximación a la responsabilidad social universitaria: la respuesta de la universidad a la sociedad”. Bordón: revista de pedagogía, No. 64: 59-81.

Nogales, Ángel. 2004. Investigación y técnicas de mercado. Madrid: ESIC.

Pacto Global de las Naciones Unidas. 2007a. "Principios para la educación responsable en gestión”. Consulta: febrero de 2015. 〈http://www.unprme.org/about-prme/index.php〉. 
---. 2007b. "Los seis principios de Principles for Responsible Management Education". Consulta: enero de 2015. 〈http://www.unprme.org/resource-docs/LOSSEISPRINCIPIOSDEPRME. pdf>.

Remolina, Gerardo. 2003. "La responsabilidad social de la universidad". Nómadas, No. 19: 239-746.

Smith, Craig. 2001. "Ethical Guidelines for Marketing Practice: A Reply to Gaski and Some Observations on the Role of Normative Marketing Ethics". Journal of Business Ethics 32, No. 1: 3-18.

UNESCO. 1998. La educación superior en el siglo XXI: misión y acción. París: UNESCO.

Universidad San Francisco de Quito (USFQ). 2013. Reglamento interno. Quito: USFQ.

Vallaeys, François. 2009. "Breve marco teórico de la responsabilidad social universitaria". Consulta: febrero de 2015. «http://rsuniversitaria.org/web/images/stories/BreveMarcoTeo delaResponsabilidadSocialUniv.pdf).

---. 2010. "Taller sobre responsabilidad social universitaria". Centro de Estudios sobre la Universidad, Universidad Autónoma de México.

Vallaeys, François, Cristina de la Cruz y Pedro Sasia. 2009. Responsabilidad social universitaria: manual de primeros pasos. México DF: McGraw-Hill.

Vallaeys, François, y Luis Carrizo. 2006. Responsabilidad social universitaria: marco conceptual, antecedentes, herramientas. Lima: Banco Interamericano de Desarrollo.

Vitell, Scott, y Joseph Paolillo. 2004. "A Cross-Cultural Study of the Antecedents of the Perceived Role of Ethics and Social Responsibility". Business Ethics 13, No. 2-3: 185199. 